Article

\title{
Impact of Bilateral Contracts on Wholesale Electricity Markets: In a Case Where a Market Participant Has Dominant Position
}

\author{
Athanasios Dagoumas \\ Energy and Environmental Policy Laboratory, Dept. of International and European Studies, \\ University of Piraeus, 18534 Piraeus, Greece; dagoumas@unipi.gr; Tel.: +30-2104142651
}

Received: 17 December 2018; Accepted: 19 January 2019; Published: 22 January 2019

check for updates

\begin{abstract}
This paper aims at tackling how the bilateral contracts affect wholesale electricity markets. It examines different levels of bilateral contracts among producers and demand aggregators, aiming to quantify their effect. In addition, it focuses on markets where bilateral contracts could be used as a tool by market participants with a dominant position. Further, the paper examined a case with asymmetrical portfolios, namely where a market participant has a dominant position as in case of Greece, aiming to investigate if bilateral contracts can be used as a tool to manipulate the market. The simulations have been done by an optimization model that provides the economic dispatch and clearing of the day-ahead electricity market. The model incorporated bilateral contracts with committed generating capacity from producers, as well as dynamic bidding strategy per market participant. Results provide useful insights on the design of electricity markets, especially in case of designing voluntary energy exchanges where a market participant has a dominant position.
\end{abstract}

Keywords: electricity market; bilateral contracts; energy prices; dominant position

\section{Introduction}

The structure of the electricity markets strongly affects competition, wholesale price signals, and the energy mix. A critical issue on the structure of the wholesale markets is which model should be adopted: From a top-down perspective, the market structure question can generally be set as selecting between a mandatory pool and a voluntary energy exchange that allows bilateral contracts among the producers and the demand aggregators. The first option is abandoned in most markets, as market liberalization favors market structures with more options for market participants. However, in the case where energy exchanges operate on a voluntary basis, there is a risk if this exchange does not have enough liquidity to provide robust price signals, especially in cases where bilateral contracts dominate the market. Moreover, depending on the generation capacity portfolio of the market participants, the existence of vertically integrated utilities with considerable shares both in production and retail markets, the level of bilateral contracts also affects the energy mix and the viability of market participants.

The impact of the bilateral contracts has been examined, but not thoroughly in the literature. Khatib and Galiana [1] examined the negotiation of bilateral contracts in electricity markets, by proposing a systematic negotiation scheme which can provide a win-win situation for the generator and the demand aggregator, as it can lead to a mutually beneficial and risk tolerable forward bilateral contracts, either physical or financial. Three papers [2-4] examined the dispatch of power networks under the existence combined pool and bilateral contracts' trading thoroughly. The paper provides results on the economic performance of market participants, aiming to help generators and retailers to select the level of pool versus bilateral trading while considering risk, economic performance, and physical constraints. 
Mari et al. [5] examined medium-term power system planning in markets with pool and bilateral contracts. The planning problem is formed with a non-convex objective function, aiming to maximize participant's revenues. In addition, it presents a heuristic procedure to tackle the nature of the problem, providing numerical results on several realistic cases with satisfactory results. Shrestha et al. [6] examined medium-term power planning with bilateral contracts, examining a Norwegian power producer that participates in the Nordic power exchange, namely Nord Pool, with the objective function to maximize the producer's revenue. Yu et al. [7] examined the negotiation of financial bilateral contracts between a generation company and a load-serving entity in wholesale electricity markets using the Nash bargaining theory. Further, the paper examines different cases and identifies situations where the two parties might fail to reach an agreement. Son et al. [8] provided a re-analysis of the Nash equilibrium bidding strategies in a bilateral electricity market, proving that, under detailed specification of the assumptions, the previously suggested Nash equilibria for the model [9] are inconsistent with the definition of Nash equilibrium. Pinto et al. [10] presented a decision support methodology for electricity market players' bilateral contract negotiations. The model is validated using real data from the Iberian market operator, MIBEL, providing results that show that the electricity market participants can improve their outcomes from bilateral contracts negotiations. Beraldi et al. [11] presented a probabilistically constrained approach for the energy procurement problem, tackling bilateral contracts as part of participants' strategy to tackle risk, through the estimation of a risk measure, the Conditional Value at Risk (CVaR).

Besides the role of bilateral contracts as an option for market participants for maximizing their revenues, the level of bilateral contracts is an important element in the design of electricity markets. Lei et al. [12] examined the electricity market design in China, including also the role of bilateral contracts, as the power exchange market of China considers bilateral contracts for long-term trading and double-sided auctions for short-term trading and the spot market. The competition of wholesale and retail markets is linked with the existence of companies with dominant positions as well as with the existence of companies with vertical integration in production, transmission/distribution, and retail sector. Market liberalization might include full-scale retail deregulation, restrictions on tariffs by dominant players, as well as unbundling of electricity generation from the transmission and distribution (T\&D) sectors. Ofuji and Tatsumi [13] provided an overview of the status of the wholesale and retail electricity markets in Japan, also describing the Electricity System Reform policy, with the aim to provide insights on whether final consumers would benefit from this process. Market reforms also considered techno-economic characteristics of the power system. Li et al. [14] identified the need for technical constraints towards a robust co-optimization of energy and reserves in real-time electricity markets Market reform might also include the adoption of other market algorithms for the clearing of the day-ahead market. Koltsaklis and Dagoumas [15] examined the adoption of the Pan-European Hybrid Electricity Market Integration Algorithm (EUPHEMIA) in the Greek electricity market, aiming to quantify the effects of different order types as well as of the strategy of a market participant with the dominant position. The paper identifies in its conclusions the need to focus more on the impact of bilateral contracts. The model developed in a recent paper [15] is further extended to incorporate the impact of bilateral contacts, which is a supplementary mechanism to the energy exchange that clears the wholesale market. The critical question that is addressed is if the liquidity of bilateral contracts among retailers and producers affects the wholesale market, namely the price signal of the energy exchange as well as the energy mix per technology and per producer. The methodology used in the paper builds on the work done at the Energy and Environmental Policy Laboratory of the University of Piraeus on unit commitment modelling [16,17], as well as on the European market clearing algorithm $[15,18]$. The simulations have been done by an optimization model that provides the economic dispatch and clearing of the day-ahead electricity market. The model incorporates bilateral contracts with committed generating capacity from producers, as well as dynamic bidding strategy per market participant. 
The literature review has revealed that the impact of bilateral contracts has not been thoroughly examined. The research focuses mainly on the maximization of profits of market participants, as well as the tackling of their risk from participating in an organized energy exchange/pool together with trading using bilateral contracts. The impact of bilateral contracts in the wholesale market has not been examined, to our knowledge. Moreover, the existence of market participants with a dominant position that could use bilateral contracts as a tool to manipulate the wholesale market has not been examined. In such cases, the level of bilateral contracts is an important decision by the regulator. Therefore, the paper contributes to the literature by quantifying the impact of bilateral contracts on wholesale electricity markets in the case where a market participant has the dominant position. Moreover, the paper contributes to the literature by incorporating dynamic bidding of the market participants, supplementary to the sensitivity analysis on bilateral contracts. The paper provides useful insights into the design of electricity markets, especially in case of designing voluntary energy exchanges where a market participant has the dominant position.

The structure of the paper is the following: The bidding strategy is described in Section 2, followed by the provision of the results in Section 3. The discussion of results is provided in Section 4, followed by the concluding remarks in Section 5 .

\section{Materials and Methods}

As mentioned in the previous sections, the simulations have been done by an optimization model that provides the economic dispatch and clearing of the day-ahead electricity market. The model developed in a recent paper [15] is further extended to incorporate the impact of bilateral contacts, which is a supplementary mechanism to the energy exchange that clears the wholesale market. In Greece, the wholesale market is currently organized as a mandatory pool, where all producers and retailers are obliged to participate. Producers submit energy offers for their power plants, while retailers submit load declarations for the consumers they represent, while traders in the interconnections submit bids for imports and exports. Public Power Corporation (PPC) is a Dominant Power Producer (DPP), owning nineteen thermal plants, fourteen lignite and five natural gas plants, while Independent Power Producers (IPPs) own five natural gas combined cycle plants. The dominant role of DPP is expected to be eliminated, to enhance competition. For environmental reasons, six lignite plants will complete their remaining operating hours within 2019 , so practically they will be decommissioned, while binding offers for the purchase of three DPP owned lignite units are expected to be submitted in early 2019. Therefore, in the examined scenarios, DPP owns five lignite plants of $1.5 \mathrm{GW}$ installed capacity and five natural gas plants of $2.6 \mathrm{GW}$, while IPPs own $0.8 \mathrm{GW}$ of lignite plants and $2.1 \mathrm{GW}$ of natural gas plants. IPPs also own $0.4 \mathrm{GW}$ of natural gas plants, characterized as an open cycle or Combined Heat and Power (CHP). Moreover, DPP own 3.2 GW of big hydro plants. Those figures show that DPP still has a dominant role in power production, besides the latest developments. Moreover, the adoption of the "target model" will allow bilateral contracts among producers and retailers, which is the focus of the paper. The decision to cap the allowed bilateral contracts is a crucial issue for decision maker by the regulator, expected to take place within 2019.

The model is developed in the Generic Algebraic Modelling System (GAMS) environment [19], supplemented by an interface for running the model and showing the results. Figure 1 provides the interface of the model developed, showing the hourly energy mix of a representative typical day. The model developed is ready for use by market participants in the real market. The model incorporates bilateral contracts with committed generating capacity from producers, as well as dynamic bidding strategy per market participant. Therefore, market participants have the option to use bilateral contracts or to participate in voluntary energy exchange. However, in the case with a participant with a dominant position, the level of allowed bilateral contracts (defined as the allowed share, in percentage, of their load in the retail market that can be met by its power units), is important for the wholesale market price and the mix of rest participants. We, therefore, examine how the level of bilateral contracts for the market participant can affect the electricity market. 
The formation of a bidding strategy is a crucial issue, as it strongly affects model outputs. Davatgaran et al. [20] proposed a model for an optimal bidding strategy of an energy hub, employing specific features, such as multi-disciplinary and flexibility. Schäfer et al. [21] presented an optimal bidding strategy from the consumption side, by examining the active participation of an aluminum electrolysis company on the primary balancing market for the provision of ancillary services on demand, such as balancing power. Afshar et al. [22] proposed an optimal bidding strategy on the production side, by examining how wind power producers can be active participants in pay-as-bid power markets. In the paper, we choose to form a dynamic bidding strategy, compared to the identification of an optimum strategy, as the focus of the paper is not the maximization of benefits for a producer or a consumer. Moreover, dynamic strategy enables a more realistic representation of market participants.



Figure 1. Interface of the model developed, showing the hourly energy mix of a representative typical day.

The impact of bilateral contracts is examined through a sensitivity analysis. The examined case is a relatively small power market, which means that unrestricted bilateral contracts could possibly lead to an energy exchange with limited liquidity. This would not lead to the provision of robust price signals. This justifies that the sensitivity analysis considers levels of bilateral contracts up to $25 \%$, which present realistic consideration by decision makers. The level of bilateral contracts is set, and the model dynamically defines the number of thermal units by the participant with the dominant position that is needed for meeting the load of the bilateral contracts. More specifically, the technical minimum of thermal units is considered, sorting units from the most expensive to the cheapest. The most expensive thermal units are selected to first, meet this bilateral contracts' load, deciding on the number and the type of units needed to operate for meeting bilateral contracts. The committed units for the satisfaction of bilateral contracts affect dynamic bidding in the energy exchange as described below.

As mentioned above, a critical assumption for the models is the formation of the bidding strategy of the market participants. More specifically, a peak saving strategy for big hydro units has been adopted, while renewables bidding was set at a very low level (e.g., $1 € / \mathrm{MWh}$ ). Concerning the interconnections and the thermal units, dynamic pricing has been adopted. Market participants are classified depending on their market share. If a market participant has a dominant position, then it adopts a different bidding strategy for their units, while participants adopt a scarcity bidding strategy for their thermal units. This scarcity pricing strategy, in general, concerns bidding at Minimum Average Variable Cost (MAVC) for their technical minimum and a "moderately aggressive" bidding for their remaining capacity, based on the "net demand" figure, as described below. 


\subsection{Dynamic Bidding of Generators and Traders in Interconnections Subsection}

\subsubsection{Generators Bidding}

The bidding strategy for the thermal units from the different market participants is done dynamically in the model, considering the capability of the market participants to adopt a scarcity pricing strategy. This is decided based in the expected "net demand" for each hour ( $t$ ):

$$
\begin{aligned}
& \text { Net_demand } d_{t}=\text { Nominal_demand }_{t}-\text { Renewables } t-H y d r o \_p r o d u c t i o n_{t}- \\
& \text { Energy_to_Bilateral_Contracts } s_{t}-\text { Tech_minimum_of_the_available_DPP_units } s_{t}- \\
& \text { Expected_Net_Imports }
\end{aligned}
$$

where:

Net_demand : Net demand during each time period $t$ (MWh)

Nominal_demand $d_{t}$ Nominal demand during each time period $\mathrm{t}(\mathrm{MWh})$

Renewables $_{t}$ : Total renewable energy generation during each time period $\mathrm{t}(\mathrm{MWh})$

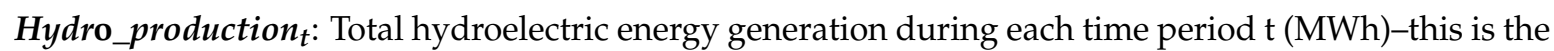
result of the initial model execution, determining the hourly allocation of the total daily hydroelectric production, based on the declared total daily production of hydroelectric units (in MWh).

Energy_to_Bilateral_Contracts ${ }_{t}$ : Committed quantity for bilateral contracts (MWh)

Tech_minimum_of_the_available_PPC_units $s_{t}$ : Sum of the technical minimum of all the available units from the Dominant Power Producer (DPP) (not included in bilateral contracts), both lignite-fired and natural gas-fired combined cycle units (MWh)

Expected_Net_Imports: Hourly net imports (positive number, while if there are net exports, they are represented by a negative number) multiplied by a specific coefficient ( $75 \%$ in our case) - this is the result of the initial model execution, determining the hourly cross-border electricity flows (net imports or exports) with each interconnected system (MWh).

Based on the hourly "net demand" estimation, the model calculates the sum of technical minimums of the available thermal units owned by independent power producers (IPPs) and compares that number (IPP_tech_min) with the resulting (Net_demand) during each hourly time period and representative day.

The following cases arise from that comparison:

If Net_demand I $_{t}<0$, then the whole capacity of IPPs is offered at their MAVC.

If Net_demand $t>0$ and Net_demand $t<I P P \_t e c h \_m i n$, then the whole capacity of IPPs is offered at their MAVC.

Net_demand $_{t}>0$ and Net_demand $t>I P P \_t e c h \_m i n$ and $N e t \_d e m a n d_{t}<I P P \_t e c h \_m i n+$ Additional capacity (above the technical minimum) of DPP units, then the model implements a sorting among the MAVC of all these units and the IPPs employ the most possible aggressive strategy (offer submission smaller by 1 cent than the MAVC of the next most expensive lignite-fired unit), if they are more economical than the DPP units (depending on the resulting merit order).

Net_demand $_{t}>0$ AND Net_demand $t>I P P \_t e c h \_m i n$ AND Net_demand $t>I P P \_t e c h \_m i n+$ Additional capacity (above the technical minimum) of DPP units, then the second step of each IPP unit (eight IPPs totally, five natural gas-fired combined cycle and three lignite-fired units) is considered to be extremely high, namely the most economical unit submits an offer at 3000/(number of available IPPs) $€ / M W h$, the second most economical unit at $2 \times 3000 /$ (number of available IPPs) $€ / M W h$, while the last available unit, which is the most expensive one, submits its remaining capacity, above its technical minimum, at $3000 € / \mathrm{MWh}$. 


\subsubsection{Bidding in Interconnections}

Based on the formation of the offers from the thermal units, in the hourly energy allocation of the hydroelectric units' contribution, whose submitted price offer equals $0 € / \mathrm{MWh}$, the model is solved again (with the same assumptions for interconnections, whose bids/offers are at the border price for their total capacity) and updated results are collected, including the system's hourly marginal prices at a daily level.

Next, the model compares each border price with the resulting marginal price (of the second run), according to the following:

- If the hourly System Marginal Price (SMP) (e.g., $50 € / \mathrm{MWh}$ ) > Border price of an interconnection (e.g., $40 € / \mathrm{MWh}$ ), then the electricity traders adapt their offers for imports between 40 and $49.999 € / M W h$, and for exports between 39.999 and $0 € / M W h$.

- If hourly SMP (e.g., $30 € / \mathrm{MWh}$ ) < Border price of an interconnection (e.g., $60 € / \mathrm{MWh}$ ), then the electricity traders adapt their offers for imports from 60 and (60 + selected coefficient) $€ / M W h$, and for exports between 59.999 and $30 € /$ MWh.

In the case of market coupling, such as the expected among the Italian and Greek power systems, bidding in interconnections is treated differently. In the case of market coupling, where power markets are co-optimized, the interconnection (i.e., Italy) is treated as a virtual plant at $500 \mathrm{MW}$, where bidding for exports from the interconnection (i.e., from Italy and imports to Greece) is set at the defined expected interconnection (Italian) price +0.001 , while imports to the interconnection (i.e., to Italy and exports from Greece) are set at the defined Italian price -0.001 .

\section{Results}

The required inputs for each scenario (simulation) concerns the hourly demand, the hourly renewables generation, the daily Mean Average Variable Cost (MAVC) of each power plant, the maintenance schedule of each power plant, the daily capacity in each interconnection, the expected hourly price in each interconnected border. The model determines the following outputs: the total hourly energy generation mix per technology type at a daily level (MWh), the hourly system's marginal price at a daily level ( $€ / \mathrm{MWh})$ and the hourly cross-border electricity flows (net imports or exports) with each interconnected system (MWh).

As mentioned above, the Greek interconnected power system has been examined. Details on its characteristics are provided in recent papers [13-16]. In Greece, the Public Power Corporation (PPC) has a dominant position with about $80 \%$ of the retail market at the end of the year 2018. Moreover, it has a diversified portfolio of power plants, including lignite-fired, combined cycle natural gas units, and big hydro units, as well as a relatively limited share in renewables capacity. The installed capacity, as well as the mix of units, compares to the independent power producers, who own only natural gas units and renewables, not operating currently any lignite or big hydro unit. However, three lignite units are expected to be purchased by IPPs within 2019, which is the situation in the examined case. This role of PPC enables the adoption of a strategy that could affect wholesale prices and mix. This was examined in a recent paper [15] concerning the adoption of a new market algorithm. The consideration of bilateral contracts as a tool for potentially influencing the wholesale market is the main focus of this paper.

The model provides several outputs, from which the most important are the hourly energy generation mix per technology type at a daily level (MWh) and the hourly system's marginal price at a daily level ( $€ / \mathrm{MWh})$.

Several scenarios have been examined. More specifically, 24 typical days (two per month) have been examined to examine four scenarios per typical day, which examine the evolution of bilateral contracts in the retail market share of PPC, taking values $10 \%, 15 \%, 20 \%$, and $25 \%$ of the load PPC represents. Therefore, 96 scenarios have been formed. The excessive number of scenarios do not allow 
for the provision of results for all scenarios. Indicative scenarios are presented that have been chosen to enable the provision of interesting and robust concluding remarks.

The weighted average annual System Marginal price for different share level of the bilateral contracts is almost identical in the examined cases, as it is estimated to be 63.11, 63.12, 63.16, 63.12, and $63.06 € / \mathrm{MWh}$ for $0 \%, 10 \%, 15 \%, 20 \%$, and $25 \%$, respectively. Figure 2 provides the average daily System Marginal price for the different typical days and the different share level of the bilateral contracts, which again depicts that results are identical for the typical days in all months.

Figure 3 provides the annual production (in TWh) per Producer and Fuel type, for different share level of the bilateral contracts $(10 \%, 15 \%, 20 \%$, and $25 \%)$. Figure 4 provides the evolution of wholesale price for different scenarios (units that participate in the bilateral contracts), different dates, and different level of bilateral contracts $(10 \%, 15 \%, 20 \%$, and $25 \%)$. The different scenarios concern a different number of thermal units from the PPC that participate in the bilateral contracts, i.e., BLOCKS_5LIG, BLOCKS_5LIG_3NG mean that five lignite or five lignite and three natural gas units participate, while the remaining capacity is bid with block orders types. On the contrary HOURLY, concern participation in the energy exchange with hourly orders. The impact of hourly and block order types has been thoroughly examined in the recent paper [15].



Figure 2. Average daily System Marginal price for the different typical days and the different share level of the bilateral contracts. 


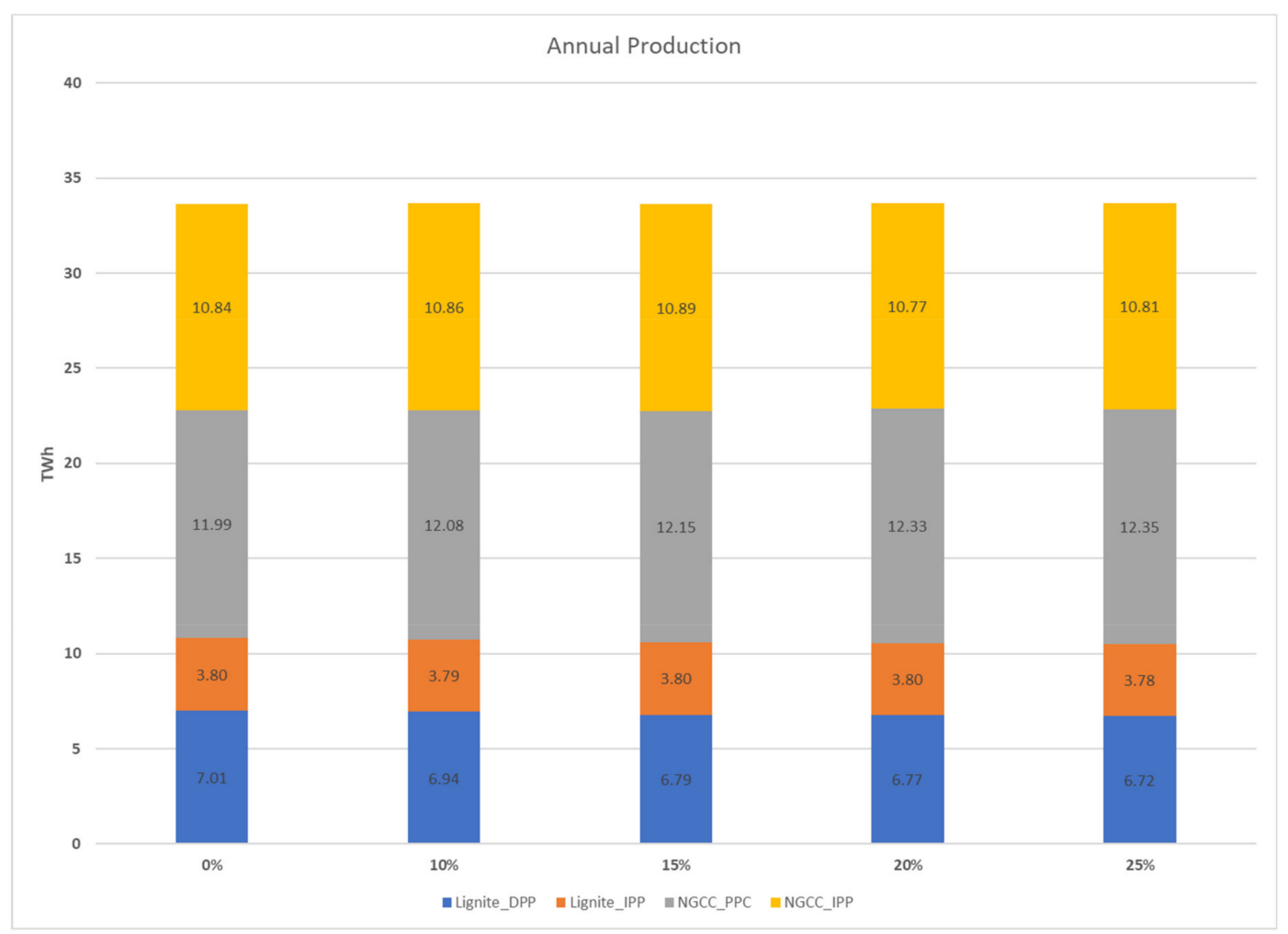

Figure 3. Annual production (in TWh) per Producer and Fuel type, for different share level of the bilateral contracts.

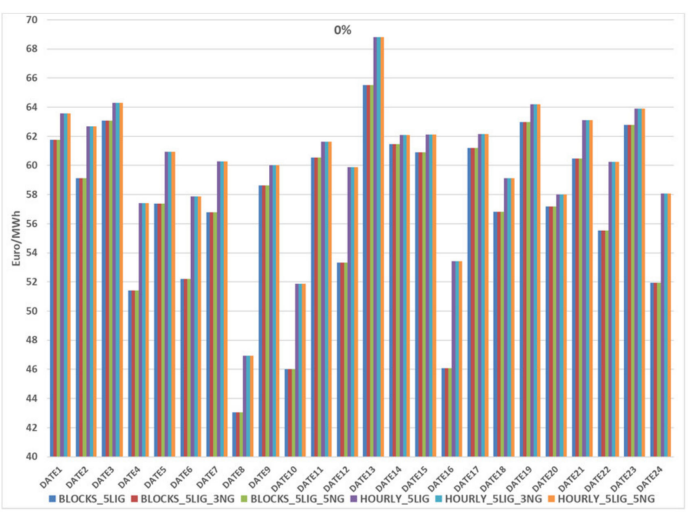

(a)

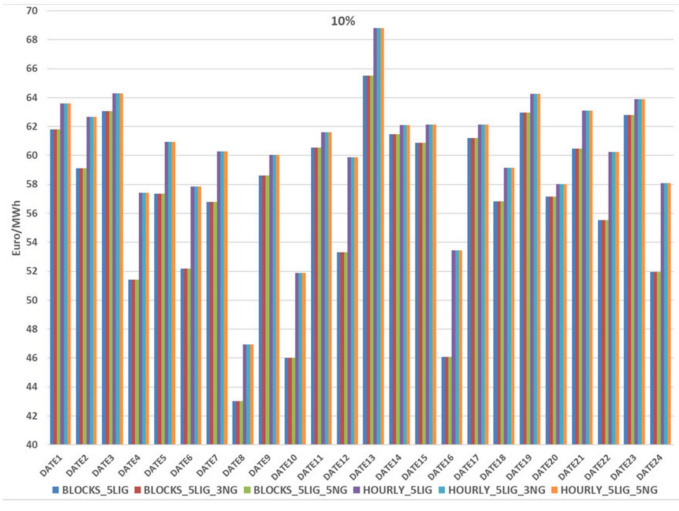

(b)

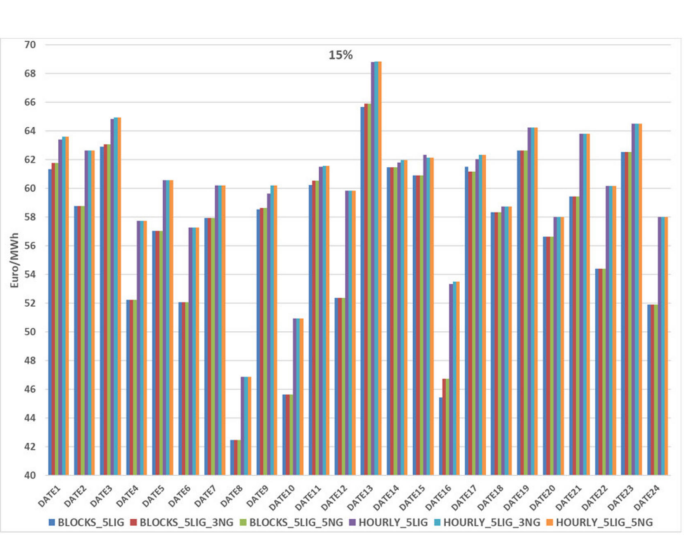

(c)

Figure 4. Cont. 


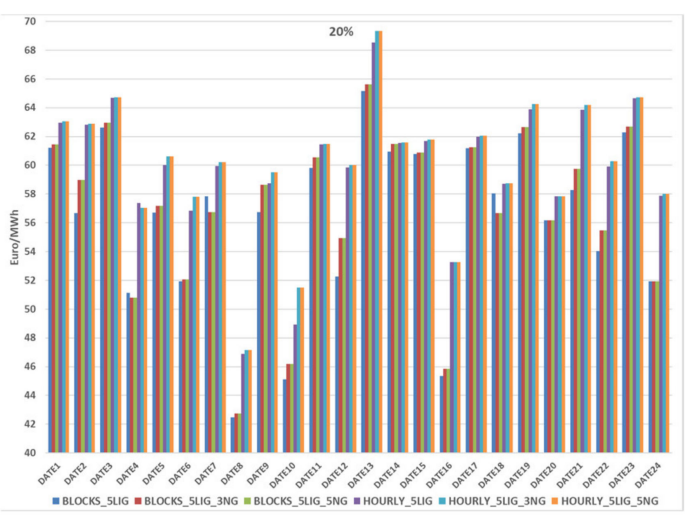

(d)

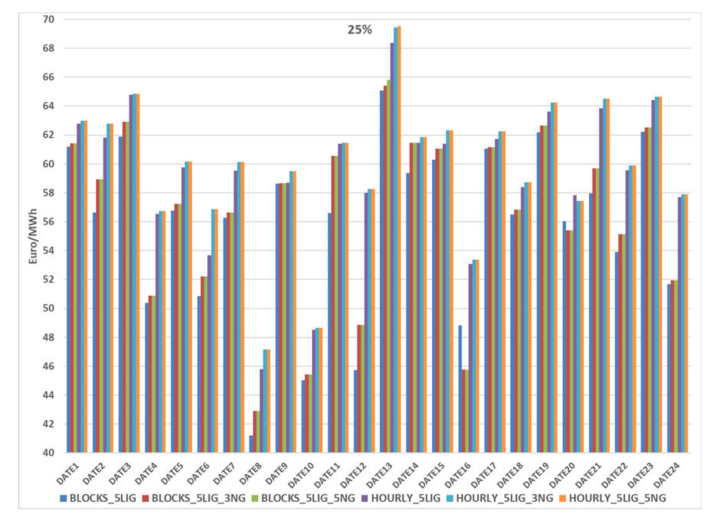

(e)

Figure 4. Wholesale price for different scenarios, dates and different level of bilateral contracts: (a) $0 \%$, (b) $10 \%$, (c) $15 \%$, (d) $20 \%$, and (e) $25 \%$.

\section{Discussion}

The adopted methodology enables the examination of the impact of bilateral contracts of the wholesale markets. The Public Power Corporation (PPC) represents about 90\% during off-peak hours (1-8), which determines the minimum load that will be covered by bilateral contracts. This leads at about three up to nine PPC thermal units to operate for meeting bilateral contracts, in case of low load $/ 10 \%$ and high load $/ 25 \%$ respectively. Besides this deviation in the committed units, the results both in the prices and energy mix are not strongly influenced. This is attributed to the fact that the new market algorithm on the voluntary energy exchange [15] enables bidding for thermal units below variable cost. The main reasoning behind that, besides the liberal assumption that every participant can adopt its strategy without constraints, is that thermal units in the European target model cannot remunerate shut-down costs through supplementary mechanisms that existed in the mandatory pool. This fact, in practice, enables PPC, as well as all thermal units, to dispatch their technical minimum with limited risk, as the remuneration of shutdown costs is difficult from the day-ahead, intra-day, and balancing markets. This is exceptionally difficult for lignite-fired units that have a high shut-down cost, as well as a long-time for the (de)synchronization of the units in case of start-up/shut-down respectively. Therefore, unless there is a specific regulatory decision on limitations in the bidding of thermal units, the dispatch of units is not strongly influenced by the introduction of bilateral contracts. In fact, the impact on the wholesale price is negligible, as the differences at the annual level are within $0.1 € / M W h$. Moreover, the non-linear evolution of SMP, as shown is Figure 2 (where the case of $15 \%$ has higher SMP compared to other cases) results from the conditions of specific dates and availability of units, which is negligible to enable the provision of a conclusion that this constitutes as an optimum level of bilateral contacts.

In the case of the energy mix, the impact is again limited, as shown in Figure 3. However, the dispatch of units is different, namely different natural gas units are dispatched at different capacities, although the sum of natural gas units is similar. This is attributed to the fact, that as the level of bilateral contracts is increased, the dominant producer PPC increases its capability to dispatch more units. Besides the lignite units, that is of high priority, due to the increased shut-down cost, that could force PPC to target at their operation at their technical minimum, the increase of bilateral contracts' level, enables the operation of higher capacity from PPC natural gas units compared to IPPs' natural gas units. However, again, the changes are relatively small but evident. Finally, the paper reveals, in alliance with the results of a recent paper [15], the impact of different order types, namely hourly and block order types. Block order types, although introduced for the needs of power producers, can lead to a reduction of wholesale price. However, as revealed in a recent paper [15] they can also lead to the evolution of wholesale price with high fluctuation, because they decrease flexibility in meeting hourly demand. 
To conclude, the implemented market strategy, as described above, allowing all thermal units to bid its technical minimum (not meeting bilateral contracts) below their Minimum Average Variable Cost (MAVC) with almost zero price, practically leads to similar power units mix. So, the level of the allowed share in bilateral contracts (10\% up to $25 \%$ ) for PPC, does not affect considerably the energy and units' mix, as well as the system marginal price. PPC would favor a higher share for bilateral contracts, but this is not a game changer in the wholesale price signal. The most important parameter is the capability of all thermal units for bidding the technical minimum below the MAVC, for avoiding the shutdown cost, which can be compensated by the wholesale market, especially for the lignite units.

\section{Conclusions}

The paper develops a model to examine the impact of bilateral contracts on wholesale electricity markets. It examines different dates, different scenarios concerning the units that participate in the bilateral contracts, and different levels of bilateral contracts. It examines a case where a utility has a dominant position. Therefore, bilateral contracts can be used as a tool for affecting the market. It incorporates dynamic bidding by the market participant, depending on their portfolio in the wholesale and retail market. Using actual data form the Greek power system, the model estimates that bilateral contracts can affect wholesale price, however, the effect is rather limited. The impact is estimated to be higher in the energy mix, where utilities with a dominant position can maximize their production, by committing expensive units in bilateral contracts and leaving their competitive units for the energy exchange. However, the impact again is limited which is justified by the fact that all thermal units are allowed to bid their technical minimum capacity (not meeting bilateral contracts) below their Minimum Average Variable Cost (MAVC), to avoid shut-down costs. This stands as the main tool of a market participants with a dominant position to affect wholesale price and power mix. Besides the fact the role of a market participant with dominant position is evident, the results both in this paper as in a recent one [15], do not reveal its capability to abuse its position, but rather the incapability of power producers to implement a scarcity pricing strategy, due to the availability of capacity, and therefore, to cover their full costs. Considering that the penetration of renewables is expected to increase considerably, due to the rapid reduction in their levelized cost of energy, this research provides insights, in alliance with the recent developments in power utilities in Europe, into not being able to compensate the full cost of their investments in thermal units.

The results provide insights that could be used by decision makers in European and other energy markets, especially in cases with asymmetrical portfolios in power generation and the existence of market participants with a dominant position. Although the imposition of different levels of cap on the bilateral contracts in case of Greece did not affect the wholesale prices and units' mix considerably, relevant analysis should be undertaken in each power market and for different scenarios, as the market power of participants is linked to the characteristics of each power market, their portfolio, the portfolio of other market participants as well as the evolution of critical factors such as fuel and $\mathrm{CO} 2$ prices.

Author Contributions: A.D. performed the research and wrote the paper.

Funding: This work has been supported by the Horizon 2020 research project INTERFACE: TSO-DSO-Consumer INTERFACE architecture to provide innovative grid services for an efficient power system (Project Grant Agreement No. 824330).

Acknowledgments: The contribution of Dr. Nikolaos Koltsaklis, senior researcher at the Energy and Environmental Policy Laboratory at the University of Piraeus, in the elaboration of the research is acknowledged.

Conflicts of Interest: The author declares no conflict of interest. 


\section{References}

1. Kathib, S.E.; Galiana, F.D. Negotiating bilateral contracts in electricity markets. IEEE Trans. Power Syst. 2007, 22, 553-562.

2. Galiana, F.D.; Kockar, I.; Franco, P.C. Combined pool/bilateral dispatch-Part I: Performance of trading strategies. IEEE Trans. Power Syst. 2002, 17, 92-99. [CrossRef]

3. Kockar, I.; Galiana, F.D. Combined pool/bilateral dispatch. II. Curtailment of firm and non-firm contracts. IEEE Trans. Power Syst. 2002, 17, 1184-1190. [CrossRef]

4. Franco, P.C.; Kockar, I.; Galiana, F.D. Combined pool/bilateral dispatch-Part III: Unbundling costs of trading services. IEEE Trans. Power Syst. 2002, 17, 1191-1198. [CrossRef]

5. Mari, L.; Nabon, N.; Pagès-Bernaus, A. Medium-term power planning in electricity markets with pool and bilateral contracts. Eur. J. Oper. Res. 2017, 260, 432-443. [CrossRef]

6. Shrestha, G.B.; Pokharel, B.K.; Lie, T.T.; Fleten, S.E. Medium term power planning with bilateral contracts. IEEE Trans. Power Syst. 2005, 20, 627-633. [CrossRef]

7. Yu, N.; Tesfatsion, L.; Liu, C.C. Financial bilateral contract negotiation in wholesale electricity markets using nash bargaining theory. IEEE Trans. Power Syst. 2012, 27, 251-267. [CrossRef]

8. Son, Y.S.; Baldick, R.; Siddiqi, S. Re-analysis of "Nash equilibrium bidding strategies in a bilateral electricity market". IEEE Trans. Power Syst. 2004, 19, 1243-1244. [CrossRef]

9. Song, H.; Liu, C.C.; Lawarrée, J. Nash equilibrium bidding strategies in a bilateral electricity market. IEEE Trans. Power Syst. 2002, 17, 73-79. [CrossRef]

10. Pinto, T.; Vale, Z.; Praça, I.; Pires, E.J.S.; Lopes, F. Decision Support for Energy Contracts Negotiation with Game Theory and Adaptive Learning. Energies 2015, 8, 9817-9842. [CrossRef]

11. Beraldi, P.; Violi, A.; Bruni, M.E.; Carrozzino, G. A Probabilistically Constrained Approach for the Energy Procurement Problem. Energies 2017, 10, 2179. [CrossRef]

12. Ofuji, K.; Tatsumi, N. Wholesale and retail electricity markets in Japan: Results of market revitalization measures and prospects for the current reform. Econ. Energy Environ. Policy 2016, 5, 31-49. [CrossRef]

13. Lei, N.; Chen, L.; Sun, C.; Tao, Y. Electricity Market Creation in China: Policy Options from Political Economics Perspective. Sustainability 2018, 10, 1481. [CrossRef]

14. Li, C.; Yun, J.; Ding, T.; Liu, F.; Ju, Y.; Yuan, S. Robust Co-Optimization to Energy and Reserve Joint Dispatch Considering Wind Power Generation and Zonal Reserve Constraints in Real-Time Electricity Markets. Appl. Sci. 2017, 7, 680. [CrossRef]

15. Koltsaklis, N.E.; Dagoumas, A.S. Policy implications of power exchanges on operational scheduling: Evaluating EUPHEMIA's market products. Energies 2018, 11, 2715. [CrossRef]

16. Koltsaklis, N.E.; Dagoumas, A.S. Transmission Expansion and Electricity Trade: A Case Study of the Greek Power System. Int. J. Energy Econ. Policy 2018, 8, 64-71.

17. Dagoumas, A.; Koltsaklis, N.; Panapakidis, I. An integrated model for risk management in electricity trade. Energy 2017, 124, 350-363. [CrossRef]

18. Koltsaklis, N.E.; Dagoumas, A.S. Incorporating unit commitment aspects to the European electricity markets algorithm: An optimization model for the joint clearing of energy and reserve markets. Appl. Energy 2018, 231, 235-258. [CrossRef]

19. GAMS Development Corporation. GAMS—A User's Guide; GAMS Development Corporation: Washington, DC, USA, May 2017; Available online: https:/ /www.gams.com/24.8/docs/userguides/GAMSUsersGuide. pdf (accessed on 15 December 2018).

20. Davatgaran, V.; Saniei, M.; Mortazavi, S.S. Optimal bidding strategy for an energy hub in energy market. Energy 2018, 148, 482-493. [CrossRef]

21. Schäfer, P.; Westerholt, H.G.; Schweidtmann, A.M.; Ilieva, S.; Mitsos, A. Model-based bidding strategies on the primary balancing market for energy-intense processes. Comput. Chem. Eng. 2019, 120, 4-14. [CrossRef]

22. Afshar, K.; Ghiasvand, F.S.; Bigdeli, N. Optimal bidding strategy of wind power producers in pay-as-bid power markets. Renew. Energy 2018, 127, 575-586. [CrossRef]

(C) 2019 by the author. Licensee MDPI, Basel, Switzerland. This article is an open access article distributed under the terms and conditions of the Creative Commons Attribution (CC BY) license (http:/ / creativecommons.org/licenses/by/4.0/). 